\title{
A NUMERICAL SOLVER FOR A NONLINEAR FOKKER-PLANCK EQUATION REPRESENTATION OF NEURONAL NETWORK DYNAMICS
}

\author{
MARÍA J. CÁCERES, JOSÉ A. CARRILLO, AND LOUIS TAO
}

\begin{abstract}
To describe the collective behavior of large ensembles of neurons in neuronal network, a kinetic theory description was developed in [13, 12], where a macroscopic representation of the network dynamics was directly derived from the microscopic dynamics of individual neurons, which are modeled by conductance-based, linear, integrate-and-fire point neurons. A diffusion approximation then led to a nonlinear Fokker-Planck equation for the probability density function of neuronal membrane potentials and synaptic conductances. In this work, we propose a deterministic numerical scheme for a Fokker-Planck model of an excitatory-only network. Our numerical solver allows us to obtain the time evolution of probability distribution functions, and thus, the evolution of all possible macroscopic quantities that are given by suitable moments of the probability density function. We show that this deterministic scheme is capable of capturing the bistability of stationary states observed in Monte Carlo simulations. Moreover, the transient behavior of the firing rates computed from the Fokker-Planck equation is analyzed in this bistable situation, where a bifurcation scenario, of asynchronous convergence towards stationary states, periodic synchronous solutions or damped oscillatory convergence towards stationary states, can be uncovered by increasing the strength of the excitatory coupling. Finally, the computation of moments of the probability distribution allows us to validate the applicability of a moment closure assumption used in [13] to further simplify the kinetic theory.
\end{abstract}

\section{INTRODUCTION}

As large-scale neuronal networks models in computational neuroscience become more common $[49,53,51,29,41,24]$, the need to develop efficient methods and effective representations for simulating and analyzing the dynamics of large-scale networks becomes urgent. The multitude of spatial and temporal scales of brain phenomena presents a challenge for model simulation and reduction. While the modularity of brain regions motivates simplification via spatial coarse-graining, irregular temporal fluctuations in the neuronal membrane potentials and the synaptic inputs [50, 46, 3, 45] suggest time-scales for temporal coarse-graining. Various theoretical approaches, based on spatial and temporal coarse-graining assumptions, have led to the development of dimensional-reduced descriptions of the network dynamics through examining a probabilistic representation of the network dynamics and deriving an evolution equation governing a probability 
density function (pdf) $[26,55,1,52,18,5,39,7,20,38,37,35,36,22,19]$. In this work, we propose an efficient numerical scheme for the simulation of a nonlinear Fokker-Planck equation representation for neuronal network dynamics.

The starting point for our description of neuronal network dynamics is a system of ordinary differential equations (ODEs) for a network of recurrently coupled, single compartment, conductance-based, linear integrate-and-fire (LIF) point neurons. In this work, we restrict the discussion to all-to-all coupled, excitatory neuronal networks. We consider the following dynamical system governing the temporal evolution of the membrane potential $V_{i}$ and the excitatory conductance $G_{i} \geq 0$ of the $i^{t h}$ neuron in a pool $\mathcal{P}_{E}$ of $N$ excitatory neurons,

$$
\begin{aligned}
\tau \frac{d V_{i}}{d t} & =-\left(V_{i}-V_{R}\right)-G_{i}\left(V_{i}-V_{E}\right) \\
\sigma_{E} \frac{d G_{i}}{d t} & =-G_{i}+\sum_{\mu} f_{E} \delta\left(t-t_{\mu}^{i}\right)+\frac{S_{E E}}{N_{E}} \sum_{j \in \mathcal{P}_{E}} \sum_{\mu} p_{j \mu}^{E} \delta\left(t-t_{\mu}^{j}\right)
\end{aligned}
$$

where $V_{E}$ is the (excitatory) reversal potential, $\tau$ is a typical leak (i.e., relaxation) time for the membrane potential, and $\sigma_{E}$ is the decay time constant of the excitatory conductance. To complete the LIF dynamical system, whenever a membrane potential, $V_{i}$, reaches the spiking threshold $\left(V_{T}\right)$, the spike time is recorded and $V_{i}$ is immediately set to the reset potential $V_{R}$.

The first sum in Equation (2) is due to incoming spikes (i.e., spikes from neurons external to the network): $t_{\mu}^{i}$ is the time of the $\mu$-th incoming spike received by the $i^{\text {th }}$ neuron. We model each incoming spike train with independent realizations of a Poisson process with rate $\nu_{0 E}(t)$. The second sum in Equation (2) describes the recurrent interaction with the other neurons in the pool via neuronal action potentials, i.e., $t_{\mu}^{j}$ is the time of the $\mu^{t h}$ spike of the $j^{t h}$ neuron. The parameter $S_{E E}$ describes the strength of network excitatory couplings. The factor of $N_{E}$ provides the overall normalization of the coupling strength. $p_{j \mu}^{E}$ is the probability of synaptic release after the arrival of each spike. We model each synaptic release as a Bernoulli process, with probability equal to $p=N_{E} / N$, that is, $p_{j \mu}^{E}=1$ with probability $p$; and 0 , otherwise.

Following $[13,12]$, by assuming that the spike trains contributing to the second sum in Equation (2) to be Poisson, the collective behavior of this network can be described in terms of a partial differential equation (PDE) for the time evolution of the probability density function (pdf), $\rho(t, v, g)$, of finding a neuron with a potential $v \in\left[V_{R}, V_{T}\right]$ and conductance $g \geq 0$ at time $t \geq 0$. A diffusive approximation, by assuming that $f_{E}$ and $S_{E E} / N_{E}$ to be small, then leads to the following Fokker-Planck equation [12]:

$$
\partial_{t} \rho=\partial_{v}\left\{\left[\left(\frac{v-V_{R}}{\tau}\right)+g\left(\frac{v-V_{E}}{\tau}\right)\right] \rho\right\}+\partial_{g}\left\{\frac{1}{\sigma_{E}}\left(g-\bar{g}_{E}(t)\right) \rho+\frac{\sigma_{g}^{2}(t)}{\sigma_{E}} \partial_{g} \rho\right\}
$$


which can be rewritten as a continuity equation

$$
\partial_{t} \rho(t, v, g)+\partial_{v} J_{V}(t, v, g)+\partial_{g} J_{G}(t, v, g)=0
$$

where the fluxes are

$$
J_{V}(t, v, g)=\left[\left(\frac{V_{R}-v}{\tau}\right)+g\left(\frac{V_{E}-v}{\tau}\right)\right] \rho(t, v, g)
$$

and

$$
J_{G}(t, v, g)=\frac{1}{\sigma_{E}}\left(\bar{g}_{E}(t)-g\right) \rho(t, v, g)-\frac{\sigma_{g}^{2}(t)}{\sigma_{E}} \partial_{g} \rho(t, v, g) .
$$

The effective drift and effective diffusivity in the conductance variable are given by:

$\bar{g}_{E}(t)=f_{E} \nu_{0 E}(t)+S_{E E} m_{E}(t) \quad$ and $\quad \sigma_{g}^{2}(t)=\frac{1}{2 \sigma_{E}}\left(f_{E}^{2} \nu_{0 E}(t)+\frac{S_{E E}^{2}}{N_{E}} m_{E}(t)\right)$

and are dependent on the average firing rate of the network $m_{E}(t)$. The network firing rate can be computed as the probability that a neuron at time $t$ achieves the voltage threshold value $V_{T}$. Thus, the firing rate is given by the probability flux at $V_{T}$, regardless of the value of the conductance, $g$, i.e.,

$$
m_{E}(t)=\int_{0}^{\infty} J_{V}\left(t, V_{T}, g\right) d g .
$$

All neurons arriving at the threshold voltage $V_{T}$ emit a spike and we assume that they instantaneously relax to their rest value $V_{R}$. Therefore, we need to supply boundary conditions for the PDE consistent with the evolution of a pdf for the trajectories of the ODE system (1)-(2) with a reset boundary condition in the membrane potential variable:

$$
\begin{array}{cc}
J_{V}\left(t, V_{T}, g\right)=J_{V}\left(t, V_{R}, g\right) & \text { for all } g \in[0, \infty) \\
J_{G}(t, v, 0)=J_{G}(t, v, \infty)=0 & \text { for all } v \in\left[V_{R}, V_{T}\right)
\end{array}
$$

That is, the flux of spiking neurons at the threshold voltage re-enters instantaneously through the reset voltage (5) and that no neuron can have negative conductances or a non-decaying conductance distribution at large conductance values (6). We point out that the $g$-boundary condition (6) can be rewritten as $\rho(t, v, 0)=0$ and $\rho(t, v, g) \rightarrow 0$ for all $v \in\left[V_{R}, V_{T}\right)$ as $g \rightarrow \infty$. Let us also remark that the $v$-boundary condition (5) on the threshold voltage implies that $\rho\left(t, V_{T}, g\right)=\rho\left(t, V_{R}, g\right)=0$ for $0 \leq g \leq g_{T}$ with the critical conductance determined by $V_{T}-V_{R}=g_{T}\left(V_{E}-V_{T}\right)$ since for excitatory neurons we have $V_{R}<V_{T}<V_{E}$. For $g \geq g_{T}$, the boundary condition (5) imposes a relation between $\rho\left(t, V_{T}, g\right)$ and $\rho\left(t, V_{R}, g\right)$. Let us finally mention that this kinetic model (3) generalizes other Fokker-Planck equations derived from some current-based LIF neuronal networks where one considers only the evolution of the voltage (see, for instance, $[7,6,30])$. 
Most studies of the behavior of neuronal networks as described by (1)-(2) through a kinetic theory $[12,44,13,11,42,33,34]$ have approached the computational simulation of this neuronal ensemble through direct Monte-Carlo simulations of the stochastic differential system (see also [47, 40], for details of the numerical schemes). Based on this Fokker-Planck equation, further dimensional reduction was obtained by a moment closure method [12] and then analyzed both numerically and theoretically [43, 28].

The direct simulation of the $(2+1)$-dimensional nonlinear Fokker-Planck equation (3) for a neuronal network has not been tackled thus far. Furthermore, a deterministic simulation has several advantages over direct Monte-Carlo simulations due to the results being noise-free, the accurate resolution of transients, and the possibility of obtaining all macroscopic quantities of interest directly from suitable moments of the pdf, $\rho(t, v, g)$. A similar strategy was adopted for obtaining highly accurate resolution of the charged particle transport in semiconductors (see $[14,15,10]$ and references therein). One of the possible drawbacks of the deterministic simulation of the Fokker-Planck equation (3) is its computational cost. However, if one wants to resolve (1)-(2) by direct Monte-Carlo simulation and obtain good statistics for distribution functions and for both dynamical transients and stationary states, the number of different realizations needed can be large, thus making the direct deterministic simulation of (3) competitive with the Monte-Carlo approach. We will comment on computational costs at the end of Section 2.

The main objectives of this work are then: to propose a highly accurate finite differences scheme for the solutions of the Fokker-Planck equation (3), to crossvalidate it against direct Monte-Carlo simulations, to analyze its performance, and to study numerically the transients of the ensemble dynamics showing the appearance of synchronous and asynchronous solutions. Concerning deterministic methods for related kinetic models, we are only aware of the results in [4]. There the authors proposed a deterministic scheme to directly solve an integrodifferential equation for a model system that included refractory effect, that is, the spiking neurons went into a refractory state which could also described by another kinetic integro-differential equation. The authors also proposed two deterministic methods: One based on a direct upwind first-order discretization of the advection derivatives together with an implicit time-stepping; the other based on splitting methods to decrease the computational time, though some time-steps are done implicitly.

In our deterministic scheme for the Fokker-Planck equation (3), we approximate the advection derivatives in voltage by finite-differences WENO (weighted essentially non-oscillatory) methods developed in $[48,25]$ for nonlinear systems of conservation laws. Since the voltage variable does not have diffusion terms (second derivatives in the voltage), a high-order method is important for the accuracy and stability for the cases where sharp fronts in the voltage develop. Both the advection and the diffusion term in the conductance variable are approximated together, as is usually done in drift-diffusion equations for semiconductors 
or in granular media models [9]; keeping good stability properties of the $g$-discretization leads to an approximation scheme known as the Chang-Cooper method. Both approximations are assembled in an ODE system which is solved by explicit 3rd-order Runge-Kutta methods as in [48]. The accurate approximation of the $v$ and $g$ derivatives allows us to use explicit time integrators with a fairly moderate CFL condition. The derivation of this scheme follows similar ideas as in work done in Boltzmann-Poisson kinetic systems for semiconductors $[14,15,10]$. Also, splitting schemes were developed for plasmas and semiconductor modelling $[17,16]$, which may be applied to the present situation for further computational savings.

After cross-validating our numerical results by direct comparisons to MonteCarlo simulations, we focus on simulating two behaviors commonly observed in neuronal network models. We first examine the appearance of bistability. Bi- and multi-stable networks have been used in models of short term working memory [54], of oculomotor integrators [27], of visual perception [32] and of neuronal populations involved in decision making [2]. We then examine a model that exhibits synchronous periodic solutions. Synchronous or oscillatory solutions have been used to model oscillations observed during cortical processing (for instance, [21, 23]). Various studies have examined how oscillatory solutions may arise in networks (see, for instance, $[1,7,6,8]$ and references therein). With our Fokker-Planck solver, we examine the transients as we increased the connectivity strength. We observe a bifurcation from asynchronous behavior or convergence towards stationary states to synchronous periodic solutions and back to convergence towards stationary solution. Finally, one advantage of the full deterministic simulation of the equation (3) is that we can compute accurately the evolution of the macroscopic moments of the pdf and their form in stationary states. Using these solutions, we validate the moment closure assumptions as proposed in [12].

The paper is structured as follows. In Section 2, we present a derivation of the deterministic scheme and compare its results to direct Monte-Carlo simulations. Section 3 details the simulation results obtained for the bistability issue, the transients synchronous versus asynchronous behavior and the validation of moment closures. We offer concluding remarks in Section 4.

\section{NUMERICAL SCHEME FOR THE KINETIC EQUATION}

Our deterministic scheme for the Fokker-Planck equation (3) is based on a fifth order WENO-finite differences approximation for the advection part in the voltage variable and an "upwind" scheme mixed with a " $\theta$-scheme", known as the Chang-Cooper method, for the advection and the diffusion term in the conductance variable. The evolution in time is done by means of a TVD thirdorder Runge-Kutta method. Both finite differences schemes (WENO and ChangCooper methods) produce very accuracy and stable approximations of derivatives on $v$ and $g$ and allow us to use explicit TVD third-order Runge-Kutta method with a fairly moderate CFL condition. 
To discretize the Fokker-Planck equation, we rewrite (3) as follows:

$$
\partial_{t} \rho(t, v, g)=-\partial_{v} J_{V}(t, v, g)+\frac{\sigma_{g}^{2}(t)}{\sigma_{E}} \partial_{g}\left\{M(g) \partial_{g}\left(\frac{\rho(t, v, g)}{M(g)}\right)\right\}
$$

where

$$
M(g)=\exp \left\{-\frac{\left|g-\bar{g}_{E}(t)\right|^{2}}{2 \sigma_{g}^{2}(t)}\right\} .
$$

and consider uniform meshes in $v$ and $g$ :

$$
\begin{array}{ll}
v_{i}=V_{R}+i \Delta v & i=0, \ldots, N_{v} \\
g_{j}=j \Delta g & j=0, \ldots, N_{g}
\end{array}
$$

where $\Delta v=\frac{V_{T}-V_{R}}{N_{v}}$ and $\Delta g=\frac{g_{\max }}{N_{g}}$. The maximum value of the conductance $g_{\max }$ is adjusted in the numerical experiments in such a way that for all $t, v$, $\rho(t, v, g) \approx 0$ for $g \geq g_{\max }$. The approximations to the point values of the solution $\rho\left(t^{n}, v_{i}, g_{j}\right)$ (denoted by $\rho_{i, j}^{n}$ ) are obtained with a dimension by dimension approximation to the derivatives on $v$ and $g$.

2.1. WENO-scheme. The advection term of the Equation (7) in the voltage variable is approximated using a fifth order conservative finite difference WENO scheme $[48,25]$. The weighted essentially non-oscillatory scheme was originally developed for hyperbolic conservation laws. These finite difference methods combine the high accuracy for the smooth parts of the evolution together with a nice treatment of possible steep fronts by locally weighting the best stencils. We remark that here, the flux in the voltage is completely linear and thus, this choice of finite differences approximation is just a simple high-order choice which adapts itself just in case of need due to the nonlinear effect in the $g$ variable and its possible transmission to the voltage variable through the drift $J_{V}(t, v, g)$. We give a summary of the WENO method below for the sake of completeness. The variable $g$ is fixed and we consider the approximation in the $v$ variable:

$$
\partial_{v} \underbrace{\left(a\left(v_{i}, g_{j}\right) \rho\left(t_{n}, v_{i}, g_{j}\right)\right)}_{J_{V}\left(t_{n}, v_{i}, g_{j}\right)} \approx \frac{1}{\Delta v}\left(\hat{h}_{i+1 / 2}-\hat{h}_{i-1 / 2}\right)
$$

where $a\left(v_{i}, g_{j}\right)=\left(\frac{V_{R}-v_{i}}{\tau}\right)+g_{j}\left(\frac{V_{E}-v_{i}}{\tau}\right)$. To explain the WENO scheme, here we assume that $a\left(v_{i}, g_{j}\right)>0$, without loss of generality (otherwise the procedure would just be mirror symmetric with respect to $i+1 / 2$ when computing $\hat{h}_{i+1 / 2}$ ). We denote by

$$
h_{i}=a\left(v_{i}, g_{j}\right) \rho\left(t_{n}, v_{i}, g_{j}\right), \quad i=-2,-1, \ldots, N_{v}+2
$$

where $j$ and $n$ are all fixed. We obtain the numerical flux by

$$
\hat{h}_{i+1 / 2}=\omega_{1} \hat{h}_{i+1 / 2}^{(1)}+\omega_{2} \hat{h}_{i+1 / 2}^{(2)}+\omega_{3} \hat{h}_{i+1 / 2}^{(3)}
$$


where $\hat{h}_{i+1 / 2}^{(m)}$ are the three third order fluxes on three different stencils given by

$$
\begin{aligned}
& \hat{h}_{i+1 / 2}^{(1)}=\frac{1}{3} h_{i-2}-\frac{7}{6} h_{i-1}+\frac{11}{6} h_{i}, \\
& \hat{h}_{i+1 / 2}^{(2)}=-\frac{1}{6} h_{i-1}+\frac{5}{6} h_{i}+\frac{1}{3} h_{i+1}, \\
& \hat{h}_{i+1 / 2}^{(3)}=\frac{1}{3} h_{i}+\frac{5}{6} h_{i+1}-\frac{1}{6} h_{i+2},
\end{aligned}
$$

The nonlinear weights $\omega_{m}$ are given by

$$
\omega_{m}=\frac{\tilde{\omega}_{m}}{\sum_{l=1}^{3} \tilde{\omega}_{l}}, \quad \tilde{\omega}_{l}=\frac{\gamma_{l}}{\left(\varepsilon+\beta_{l}\right)^{2}},
$$

with $\varepsilon=10^{-6}$, the linear weights $\gamma_{l}$ given by

$$
\gamma_{1}=\frac{1}{10}, \quad \gamma_{2}=\frac{3}{5}, \quad \gamma_{3}=\frac{3}{10},
$$

and the smoothness indicators $\beta_{l}$ given by

$$
\begin{aligned}
& \beta_{1}=\frac{13}{12}\left(h_{i-2}-2 h_{i-1}+h_{i}\right)^{2}+\frac{1}{4}\left(h_{i-2}-4 h_{i-1}+3 h_{i}\right)^{2} \\
& \beta_{2}=\frac{13}{12}\left(h_{i-1}-2 h_{i}+h_{i+1}\right)^{2}+\frac{1}{4}\left(h_{i-1}-h_{i+1}\right)^{2} \\
& \beta_{3}=\frac{13}{12}\left(h_{i}-2 h_{i+1}+h_{i+2}\right)^{2}+\frac{1}{4}\left(3 h_{i}-4 h_{i+1}+h_{i+2}\right)^{2} .
\end{aligned}
$$

As usual with this kind of schemes, some ghost points are necessary in the mesh, which are chosen to impose numerically the boundary conditions (5). This condition for the flux in $v$ means that for every $g$ if $a\left(t, V_{T}, g\right)<0$ then $J_{V}\left(t, V_{T}, g\right)=0$ and consequently $J_{V}\left(t, V_{R}, g\right)=0$, otherwise $J_{V}\left(t, V_{R}, g\right)=J_{V}\left(t, V_{T}, g\right)$. Therefore, for $n, j$ fixed, the values at the ghost points are defined as: if $a\left(t_{n}, V_{T}, g_{j}\right)<0$ then $h_{-i}=0$ and $h_{N_{v}+i}=0$ for $i=1,2,3$, otherwise if $a\left(t_{n}, V_{T}, g_{j}\right) \geq 0$ thus $h_{-i}=h_{N_{v}+1}$ and $h_{N_{v}+i}=h_{N_{v}+1}$ for $i=1,2,3$, using the notation (8).

2.2. Chang-Cooper Method. For the derivatives of the conductance variable in the Equation (7) we follow the Chang-Cooper method as considered in [9]. Originally, the Chang-Cooper method was designed to preserve the equilibrium state of the Fokker-Plank equation. Moreover, this scheme is also an entropy decay preserving method. This feature is important to get accurate behavior for large times of the discretization scheme in $g$. In fact, it ensures that the maxwellian equilibria $M(g)$ are preserved for the discretized scheme in the absence of the external time dependent inputs and for homogeneous data in $v$. In the jargon of the numerical conservation law community, this property is known as well-balanced. Moreover, it keeps the associated Liapunov functional (called 
entropy in statistical mechanics) decreasing in time for the scheme. As in the voltage variable we approximate the term of conductance using finite differences:

$$
\partial_{g} \frac{\sigma_{g}^{2}\left(t_{n}\right)}{\sigma_{E}}\left\{M\left(g_{j}\right) \partial_{g}\left(\frac{\rho\left(t_{n}, v_{i}, g_{j}\right)}{M\left(g_{j}\right)}\right)\right\} \approx \frac{F_{j+1 / 2}-F_{j-1 / 2}}{\Delta g}
$$

where, for $n$ and $i$ fixed, the numerical flux is

$$
F_{j+1 / 2}=\frac{\sigma_{g}^{2}\left(t_{n}\right)}{\sigma_{E} \Delta g} \tilde{M}_{j+1 / 2}\left(\frac{\rho_{j+1}}{M_{j+1}}-\frac{\rho_{j}}{M_{j}}\right)
$$

and

$$
\tilde{M}_{j+1 / 2}=\frac{M_{j} M_{j+1}}{M_{j+1}-M_{j}}\left(\ln \left(M_{j+1}\right)-\ln \left(M_{j}\right)\right)
$$

is a value between $M_{j}$ and $M_{j+1}$. We have used the following notations: $M_{j}=$ $M\left(g_{j}\right)$ and $\rho_{j}=\rho\left(t_{n}, v_{i}, g_{j}\right)$. After some computations, see [9] for details, $F_{j+1 / 2}$ can be rewritten as:

$$
F_{j+1 / 2}=\frac{\sigma_{g}^{2}(t)}{\sigma_{E} \Delta g}\left(\rho_{j+1}-\rho_{j}\right)+\frac{\sigma_{g}^{2}(t) \omega}{\sigma_{E} \Delta g}\left(\theta \rho_{j}+(1-\theta) \rho_{j+1}\right)
$$

where $\omega=\ln \left(M_{j} / M_{j+1}\right)$ and $\theta=\frac{1}{\omega}-\frac{1}{e^{\omega}-1}$. Now we observe, as we announced above, that (9) is an "upwind" scheme, mixed with a " $\theta$-scheme". To conclude with the description of the numerical approximation in $g$, we remark that the ghost flux for $j=-1$ and $j=N_{g}+1$ are considered null, taking into account the boundary condition for $g$ flux in (6).

2.3. TVD $3^{\text {rd }}$-order RK. The evolution in time is implemented by means of the third order TVD Runge-Kutta method as in [48]:

$$
\begin{aligned}
\rho^{(1)} & =\rho^{n}+\Delta t L\left(\rho^{n}, t^{n}\right) \\
\rho^{(2)} & =\frac{3}{4} \rho^{n}+\frac{1}{4} \rho^{(1)}+\frac{1}{4} \Delta t L\left(\rho^{(1)}, t^{n}+\Delta t\right) \\
\rho^{n+1} & =\frac{1}{3} \rho^{n}+\frac{2}{3} \rho^{(2)}+\frac{2}{3} \Delta t L\left(\rho^{(2)}, t^{n}+\frac{1}{2} \Delta t\right),
\end{aligned}
$$

where $L$ is the approximation to the advection and diffusion terms:

$$
L(\rho(t, g, v), t) \approx-\partial_{v} J_{V}(t, v, g)+\frac{\sigma_{g}^{2}(t)}{\sigma_{E}} \partial_{g}\left\{M(g) \partial_{g}\left(\frac{\rho(t, v, g)}{M(g)}\right)\right\}
$$

and $\Delta t$ is the time step, which is conditioned on the following CFL restriction since an explicit scheme is considered:

$$
\Delta t \leq C F L \min \left\{\frac{\Delta v}{\max \left|a\left(v_{i}, g_{j}\right)\right|}, \frac{(\Delta g)^{2}}{\frac{\sigma_{g}^{2}\left(t_{n}\right)}{\sigma}+\Delta g \max \left|F_{j+1 / 2}\right|}\right\}
$$


where we recall that $a(v, g)=\left(\frac{V_{R}-v}{\tau}\right)+g\left(\frac{V_{E}-v}{\tau}\right)$. Due to the accurate approximations of the WENO-scheme and the Chang-Cooper method of the fluxes, the CFL condition does not yield restrictive time-stepping.

Finally, since the system is nonlinear due to the firing rate:

$$
m_{E}(t)=\int_{0}^{\infty} J_{V}\left(t, V_{T}, g\right) d g
$$

which need to be incorporated in a self-consistent fashion, at each time step, we approximate $m_{E}$ by the composite mid-point rule and re-inject it in the next step. In our simulations we consider as initial data the product of two different Maxwellian functions in $g$ and $v$ both normalized to be probability densities, i.e., unit numerical mass. For the bistable systems we proceed in a different way, as we explain below in Section 3.

2.4. Comparison to Monte-Carlo Simulations. We solve the set of Equations (1)-(2) with a modified second-order Runge-Kutta scheme [47] with a numerical time-step of $0.01 \mathrm{~ms}$. In a few cases, runs with a numerical time-step of 0.001 ms were performed and no significant quantitative differences were observed. We note that Equations (1)-(2) are already written in reduced-dimensional units, in which only time has dimensions, in ms; see, for instance, [31]. We set the network pool size to be $N=100,000$ neurons, each one connected to $N_{E}=100$ neurons in the direct Monte-Carlo simulations. The parameters of the system are as follows: $\tau=20 \mathrm{~ms}, \sigma_{E}=3 \mathrm{~ms}, V_{E}=14 / 3, V_{T}=1, V_{R}=0$ and we set equal the rest and reset potentials. We set $f_{E}=10 \mathrm{~ms}$ and $S_{E E}=0.05$ for our comparison between the Monte-Carlo and Fokker-Planck solvers.

We compare our numerical Fokker-Planck solver to direct Monte Carlo simulations via the following examples, going from stationary to non-stationary cases.

Stationary case. Case (A) We consider a network in which the rate of input Poisson process $\nu_{0 E}(t)=A$ with constant $A \in[1000,1500]$.

Figure 1 compares the network firing rate versus mean $g_{i n p}=f_{E} \nu_{0 E}$ between the Monte-Carlo and the Fokker-Planck simulations. We observe very good agreement between the simulations. In Figure 5 we compare the pdfs between the two simulations. We fix $A=1400$ and we display marginal probability distribution functions $\rho_{v}$ and $\rho_{g}$ for the stationary solutions. Again we observe that the numerical results from the two solvers are in excellent agreement. We would like to point out that for the results from the deterministic Fokker-Planck simulations, the distribution functions $\rho_{v}$ and $\rho_{g}$ were obtained by numerical integrations of $\rho$, therefore there was not need for us to consider derived Fokker-Planck systems (say, by assuming a closure hypothesis) for $\rho_{v}$ or for $\rho_{g}$. Consequently, we obtain the time evolution of these distribution functions as we observe in Figure $6, \rho_{g}$ at times $0.05,0.1$ and 0.15 is the same (steady state), however, at time $0.05 \rho_{v}$ has not still achieved the stationary state. 
Non stationary case. We also analyze cases where the rate of Poisson process is not constant in time. We consider the two following cases:

- Case B: The input Poisson process has a rate that depends on time continuously

$$
\begin{array}{ll} 
& \nu_{0 E}(t)=A(1+\epsilon \sin (\omega t)) \\
\text { B1: } & A=1500, \epsilon=300, \omega=8 \pi \\
\text { B2: } & A=1000, \epsilon=200, \omega=8 \pi
\end{array}
$$

- Case $C$ : The input Poisson process has a rate that has a discrete jump in time

$$
\begin{aligned}
& \nu_{0 E}(t)=A \theta\left(t-t_{0}\right)+B, \quad \theta \text { being the Heaviside function } \\
& A=500, B=1000, t_{0}=1
\end{aligned}
$$

Figures 2 and 3 compares the time evolution of the firing rate for the Case $B$ (B1 and B2), whereas Figure 4 shows the firing rate in the Case $C$. Comparing these figures we observe how the shape of the external input is reflected in the evolution of the firing rate.

We note that simulating accurately ensembles of 100,000 LIF neurons to get good statistics took 20-50 times longer than the corresponding Fokker-Planck equation in all cases. The difference is more pronounced in the cases where we are interested in the dynamical transients (for instance, in Case C), when a larger number of ensembles (and/or realizations) are needed for a accurate resolution of the pdfs.

\section{Simulation Results}

Here we illustrate the use of our numerical Fokker-Planck solver by examining the solutions to the following problems, produced by different choices of input forcing, $\nu_{0 E}$ : stationary case with a unique steady state, non-stationary solutions and a case exhibiting bistability. Finally, using our numerical solver we check the closure condition (10) in a variety of circumstances.

3.1. Bistability. Bistability is typical of systems with strong excitatory feedback. Even in situations as in Case $A$ above, there are circumstances, when the recurrent excitatory coupling is sufficiently strong, that exhibit two distinct steady state solutions (with different firing rates) for the same input forcing. For instance, let us consider an example with the following parameters: $f_{E}=1 / 200 \mathrm{~s}, S_{E E}=0.2, N_{E}=200$ and $\sigma_{E}=2 \mathrm{~ms}$. Figure 7 shows bistability in the firing rate vs. input strength diagram. Here the two different branches have different firing rates and can be distinguished by the choice of initial data. To obtain this firing rate diagram we fit for an input of $f_{E} \nu_{0 E}=11$ as initial data the product of two different Maxwellian functions: one for the variable $g$ 
and other one for the variable $v$, each normalized to have unit numerical integral (since $\rho(v, g)$ is a probability distribution). Then the stationary solution of this problem is considered as initial data for the system with $f_{E} \nu_{0 E}=10.9$, whose stationary state is taken as initial data for the case $f_{E} \nu_{0 E}=10.8$ and and so on, to obtain the firing rates of the top branch. However, to obtain the lower branch, it is not necessary to produce in a similiar fashion, since this branch appears considering the same initial data for all the input $f_{E} \nu_{0 E}$.

In Figures 8-9, we display temporal evolution (of network firing rates) and the (eventual) steady-state pdf for $f_{E} \nu_{0 E}=10.8$ which is in the parameter regime exhibiting bistability. Figure 8 shows the evolution of the firing rate and the stationary solution for the lower branch, whereas Figure 9 shows the same for the upper branch. Note that we needed to start with initial data with high firing rates to access the upper branch. We also remark that there are damped, oscillatory transients in the latter case. Let us next turn to the development of oscillatory solutions.

3.2. Synchronous versus Asynchronous solutions. In Figure 10 we exhibit the firing rate for $f_{E} \nu_{0 E}=11$ where there is no bistability. (Thus any initial data will eventually approach the unique, high firing rate, steady state solution.) The damped oscillatory transients may be the signature of oscillatory solutions that are nearby in parameter space. Therefore, let us now consider the following case, $S_{E E}=0.15, N_{E}=500, f_{E}=0.002 \mathrm{~s}$ and $f_{E} \nu_{0 E}=12$, where firing rate is periodic in time; see Figure 11. In fact, if we fix $N_{E}=500, f_{E}=0.002 \mathrm{~s}$ and $f_{E} \nu_{0 E}=12$ and vary $S_{E E}$, we obtain Figure 12, which shows that there is a range in the excitatory coupling strength, $S_{E E}$, approximately, between 0.13 and 0.17 where periodic solutions appear (and outside of this interval oscillations do not happen). For small $S_{E E}$, solutions go quickly to the steady state but for slightly larger values over this interval $\left(S_{E E} \geq 0.17\right)$, the solution reaches the steady state in an oscillatory way, starting with huge oscillations which vanish after some time.

In the case where $0.13 \leq S_{E E} \leq 0.17$ and periodic solutions appear, we also observe that maximum firing rate increases when $S_{E E}$ is increased. Figure 12 shows that the maximum value for $S_{E E}=0.13$ is around $250 \mathrm{spikes} / \mathrm{sec}$, while for $S_{E E}=0.14$ is around 350 spikes/sec, for $S_{E E}=0.16$ is less than 500 spikes/sec and for $S_{E E}=0.17$ is more than 500 spikes/sec. At the same time, as we increase $S_{E E}$ the frequency of oscillations increases also. However, eventually the oscillations damp out again and steady state solutions are obtained and asynchronous behavior is observed again (see last panel of Figure 12).

3.3. Validation of moment closure assumptions. Equation (3) is a nonlinear $(2+1)$ dimensional problem and requires a numerical solver, one of which has been the subject of this paper. To simplify the mathematical representation further, one can project out the $g$ variable by defining the conditional moments 
$\mu_{n}(v)=\int_{0}^{\infty} g^{n} \rho(g \mid v) d g$, for $n=1,2, \ldots$ where $\rho(g \mid v)$ is the (conditional) pdf of $g$ given $v$ and can be computed from $\rho(t, v, g)=\rho(g \mid v) \rho_{v}(t, v)$. However, this will lead to a hierarchy of moment equations, where the dynamical equations for lower order moments depend on higher order moments. To close this hierarchy, in [12] the authors postulated a closure condition

$$
\Sigma^{2}(t, v)=\sigma_{g}^{2}(t)
$$

for the conditional variance, $\Sigma^{2}(t, v) \equiv \mu_{2}(t, v)-\mu_{1}^{2}(t, v)$, and derived a system of two $(1+1)$ dimensional PDEs for $\rho_{v}(t, v)=\int_{0}^{\infty} \rho(t, v, g) d g$ and $\mu_{1}(t, v)$ :

$$
\begin{aligned}
\partial_{t} \rho_{v}(t, v)= & \partial_{v}\left\{\left[\left(\frac{v-V_{R}}{\tau}\right)+\mu_{1}(t, v)\left(\frac{v-V_{E}}{\tau}\right)\right] \rho_{v}(t, v)\right\} \\
\partial_{t} \mu_{1}(t, v)= & -\frac{1}{\sigma_{E}}\left[\mu_{1}(t, v)-\bar{g}(t)\right]+\frac{\sigma_{g}^{2}(t)}{\rho_{v}(t, v)} \partial_{v}\left[\left(\frac{v-V_{E}}{\tau}\right) \rho_{v}(t, v)\right] \\
& +\left[\left(\frac{v-V_{R}}{\tau}\right)+\mu_{1}(t, v)\left(\frac{v-V_{E}}{\tau}\right)\right] \partial_{v} \mu_{1}(t, v) .
\end{aligned}
$$

With our numerical scheme we can validate the closure assumption $\Sigma^{2}(t, v)=$ $\sigma_{g}^{2}(t)(10)$ since we can easily compute moments of the distribution function. Figure 13 compares the conditional variance $\Sigma^{2}(t, v)$ with the variance $\sigma_{g}^{2}(t)$ for different values of $A$ in Case $A$ where unique, steady state solutions exist. We note that, in the steady state, the closure condition is satisfied, on average, away from the $v$-boundaries. More precisely, the maximum $L^{\infty}$ difference of the two variances occurs at $v=1$ (for all but the briefest of initial transients). In Figure 14 we observe similar behavior in the case of where bistable solutions exist. In general, we observe that the closure assumption is reasonable for most values of $v$, away from the boundaries.

\section{Conclusions}

Our numerical Fokker-Planck solver is an efficient and accurate way of simulating the effective dynamics of a large-scale LIF neuronal network. This deterministic representation of the dynamics at the macroscopic level allows us to efficiently track the temporal evolution of the pdfs and to obtain any macroscopic quantities of the network dynamics. In this work, we have validated our numerical scheme by comparing it with stochastic Monte Carlo simulations. We have also used our numerical solver to analyze a series of numerical examples. In future work, we will extend our numerical scheme to networks with both excitatory and inhibitory couplings and to networks with spatial dependencies. This future project is not a direct nor obvious modification of the scheme developed in this paper: the boundary conditions in voltage are more complicated when networks with both excitatory and inhibitory couplings are considered, more variables 
(and thus more equations) are needed, the spatial dependence has to be clarified at the level of the Fokker-Plank equation, etc. Therefore, the work included in this paper is an essential step towards simulating realistic, large-scale neuronal network behavior.

\section{REFERENCES}

[1] L. Abbott and C. van Vreeswijk. Asynchronous states in networks of pulse-coupled oscillators. Phys. Rev. E, 48:1483-1490, 1993.

[2] L. Albantakis and G. Deco. The encoding of alternatives in multiple-choice decision making. Proc Natl Acad Sci U S A, 106(25):10308-10313, Jun 2009.

[3] J. Anderson, I. Lampl, D. Gillespie, and D. Ferster. The contribution of noise to contrast invariance of orientation tuning in cat visual cortex. Science, 290:1968-1972, 2000.

[4] F. Apfaltrer, C. Ly, and D. Tranchina. Population density methods for stochastic neurons with realistic synaptic kinetics: Firing rate dynamics and fast computational methods. Network: Computation in Neural Systems, 17:373-418, 2006.

[5] G. Barna, T. Grobler, and P. Erdi. Statistical model of the hippocampal ca3 region, ii. the population framework: model of rhythmic activity in ca3 slice. Biol. Cybern., 79:309-321, 1998.

[6] N. Brunel. Dynamics of sparsely connected networks of excitatory and inhibitory spiking networks. J. Comp. Neurosci., 8:183-208, 2000.

[7] N. Brunel and V. Hakim. Fast global oscillations in networks of integrate-and-fire neurons with long fiting rates. Neural Computation, 11:1621-1671, 1999.

[8] N. Brunel and X.-J. Wang. What determines the frequency of fast network oscillations with irregular neural discharge? I. synaptic dynamics and excitation-inhibition balance. J. Neurophysiol., 90:415-430, 2003.

[9] C. Buet, S. Cordier, and V. Dos Santos. A conservative and entropy scheme for a simplified model of granular media. Transport Theory Statist. Phys., 33(2):125-155, 2004.

[10] M. J. Cáceres, J. A. Carrillo, I. M. Gamba, A. Majorana, and C.-W. Shu. Deterministic kinetic solvers for charged particle transport in semiconductor devices. In Transport phenomena and kinetic theory, Model. Simul. Sci. Eng. Technol., pages 151-171. Birkhäuser Boston, Boston, MA, 2007.

[11] D. Cai, L. Tao, and D. W. McLaughlin. An embedded network approach for scale-up of fluctuation-driven systems with preservation of spike information. PNAS, 101:14288-14293, 2004.

[12] D. Cai, L. Tao, A. V. Rangan, and D. W. McLaughlin. Kinetic theory for neuronal network dynamics. Commun. Math. Sci., 4(1):97-127, 2006.

[13] D. Cai, L. Tao, M. J. Shelley, and D. W. McLaughlin. An effective kinetic representation of fluctuation-driven neuronal networks with application to simple and complex cells in visual cortex. PNAS, 101:7757-7762, 2004.

[14] J. A. Carrillo, I. M. Gamba, A. Majorana, and C.-W. Shu. A WENO-solver for the transients of Boltzmann-Poisson system for semiconductor devices: performance and comparisons with Monte Carlo methods. J. Comput. Phys., 184(2):498-525, 2003.

[15] J. A. Carrillo, I. M. Gamba, A. Majorana, and C.-W. Shu. 2D semiconductor device simulations by WENO-Boltzmann schemes: efficiency, boundary conditions and comparison to Monte Carlo methods. J. Comput. Phys., 214(1):55-80, 2006.

[16] J. A. Carrillo, A. Majorana, and F. Vecil. A semi-lagrangian deterministic solver for the semiconductor boltzmann-poisson system. Commun. Comput. Phys., 2:1027-1054, 2007. 
[17] J. A. Carrillo and F. Vecil. Nonoscillatory interpolation methods applied to Vlasov-based models. SIAM J. Sci. Comput., 29(3):1179-1206 (electronic), 2007.

[18] T. Chawanya, A. Aoyagi, T. Nishikawa, K. Okuda, and Y. Kuramoto. A model for feature linking via collective oscillations in the primary visual cortex. Biol. Cybern., 68:483-90, 1993.

[19] N. Fourcaud and N. Brunel. Dynamics of the firing probability of noisy integrate-and-fire neurons. Neural Comp., 14:2057-2110, 2002.

[20] W. Gerstner. Population dynamics of spiking neurons: Fast transients, asynchronous states, and locking. Neural Comp., 12:43-89, 2000.

[21] C. M. Gray and W. Singer. Stimulus-specific neuronal oscillations in orientation columns of cat visual cortex. Proc Natl Acad Sci U S A, 86(5):1698-1702, 1989.

[22] E. Haskell, D. Nykamp, and D. Tranchina. Population density methods for large-scale modeling of neuronal networks with realistic synaptic kinetics: cutting the dimension down to size. Network: Compt. Neural. Syst, 12:141-174, 2001.

[23] J. A. Henrie and R. Shapley. Lfp power spectra in v1 cortex: the graded effect of stimulus contrast. J Neurophysiol, 94(1):479-490, 2005.

[24] E. M. Izhikevich and G. M. Edelman. Large-scale model of mammalian thalamocortical systems. Proc Natl Acad Sci U S A, 105(9):3593-3598, Mar 2008.

[25] G.-S. Jiang and C.-W. Shu. Efficient implementation of weighted ENO schemes. J. Comput. Phys., 126(1):202-228, 1996.

[26] B. Knight. Dynamics of encoding in a populaton neurons. J. Gen. Physiol., 59:734-766, 1972.

[27] A. Koulakov, S. Raghavachari, A. Kepecs, and J. Lisman. Model for a robust neural integrator. Nat. Neurosci., 5:775-782, 2002.

[28] C. Ly and D. Tranchina. Critical analysis of dimension reduction by a moment closure method in a population density approach to neural network modeling. Neural Comput., 19(8):2032-2092, 2007.

[29] J. Marino, J. Schummers, D. C. Lyon, L. Schwabe, O. Beck, P. Wiesing, K. Obermayer, and M. Sur. Invariant computations in local cortical networks with balanced excitation and inhibition. Nature Neurosci., 8:194-201, 2005.

[30] M. Mattia and P. Del Giudice. Population dynamics of interacting spiking neurons. Phys. Rev. E, 66:051917, 2002.

[31] D. McLaughlin, R. Shapley, M. Shelley, and J. Wielaard. A neuronal network model of macaque primary visual cortex (V1): Orientation selectivity and dynamics in the input layer 4C $\alpha$. Proc. Natl. Acad. Sci. USA, 97:8087-8092, 2000.

[32] R. Moreno-Bote, J. Rinzel, and N. Rubin. Noise-induced alternations in an attractor network model of perceptual bistability. J. Neurophysiol., 98:1125-1139, 2007.

[33] K. Newhall, G. Kovačič, P. Kramer, A. V. Rangan, and D. Cai. Cascade-induced synchrony in stochastically-driven neuronal networks. preprint, 2010.

[34] K. Newhall, G. Kovačič, P. Kramer, D. Zhou, A. V. Rangan, and D. Cai. Dynamics of current-based, poisson driven, integrate-and-fire neuronal networks. Comm. in Math. Sci., 8:541-600, 2010 .

[35] D. Nykamp and D. Tranchina. A population density method that facilitates large-scale modeling of neural networks: Analysis and application to orientation tuning. J. of Computational Neuroscience, 8:19-50, 2000.

[36] D. Nykamp and D. Tranchina. A population density method that facilitates large-scale modeling of neural networks: Extension to slow inhibitory synapses. Neural Computation, 13:511-546, 2001.

[37] A. Omurtag, E. Kaplan, B. Knight, and L. Sirovich. A population approach to cortical dynamics with an application to orientation tuning. Network, 11:247-260, 2000. 
[38] A. Omurtag, B. Knight, and L. Sirovich. On the simulation of large populations of neurons. J. of Comp. Neurosci., 8:51-63, 2000.

[39] J. Pham, K. Pakdaman, J. Champagnat, and J. Vibert. Activity in sparsely connected excitatory neural networks: effect of connectivity neural networks. Neural Networks, 11:415434, 1998.

[40] A. V. Rangan and D. Cai. Fast numerical methods for simulating large-scale integrateand-fire neuronal networks. J. Comput. Neurosci., 22(1):81-100, 2007.

[41] A. V. Rangan, D. Cai, and D. W. McLaughlin. Modeling the spatiotemporal cortical activity associated with the line-motion illusion in primary visual cortex. PNAS, 102:18793$18800,2005$.

[42] A. V. Rangan, D. Cai, and D. W. McLaughlin. Quantifying neuronal network dynamics through coarse-grained event trees. PNAS, 105:10990-10995, 2008.

[43] A. V. Rangan, D. Cai, and L. Tao. Numerical methods for solving moment equations in kinetic theory of neuronal network dynamics. J. Comput. Phys., 221(2):781-798, 2007.

[44] A. V. Rangan, G. Kovačič, and D. Cai. Kinetic theory for neuronal networks with fast and slow excitatory conductances driven by the same spike train. Phys. Rev. E (3), 77(4):041915, 13, 2008.

[45] M. Rudolph and A. Destexhe. Characterization of subthreshold voltage fluctuations in neuronal membranes. Neural Comp., 15:2577-2618, 2003.

[46] M. Shadlen and W. Newsome. The variable discharge of cortical neurons: implications for connectivity, computation and information coding. J Neurosci, 18:3870-3896, 1998.

[47] M. Shelley and L. Tao. Efficient and accurate time-stepping schemes for integrate-and-fire neuronal networks. J. Comp. Neurosci., 11:111-119, 2001.

[48] C.-W. Shu and S. Osher. Efficient implementation of essentially nonoscillatory shockcapturing schemes. J. Comput. Phys., 77(2):439-471, 1988.

[49] D. C. Somers, S. B. Nelson, and M. Sur. An emergent model of orientation selectivity in cat visual cortical simple cells. J Neurosci, 15(8):5448-5465, 1995.

[50] E. Stern, A. Kincaid, and C. Wilson. Spontaneous subthreshold membrane potential fluctuations and action potential variability of rat corticostriatal and striatal neurons in vivo. J. Neurophysiol., 77:1697-1715, 1997.

[51] L. Tao, M. Shelley, D. McLaughlin, and R. Shapley. An egalitarian network model for the emergence of simple and complex cells in visual cortex. Proc Natl Acad Sci U S A, 101(1):366-371, 2004.

[52] A. Treves. Mean field analysis of neuronal spike dynamics. Network, 4:259-284, 1993.

[53] T. Troyer, A. Krukowski, N. Priebe, and K. Miller. Contrast invariant orientation tuning in cat visual cortex with feedforward tuning and correlation based intracortical connectivity. J. Neurosci., 18:5908-5927, 1998.

[54] X. Wang. Synaptic basis of cortical persistent activity: The importance of NMDA receptors to working memory. J. Neurosci., 19:9587-9603, 1999.

[55] W. Wilbur and J. Rinzel. A theoretical basis for large coefficient of variation and bimodality in neuronal interspike interval distributions. J. Theor. Biol, 105:345-368, 1983. 


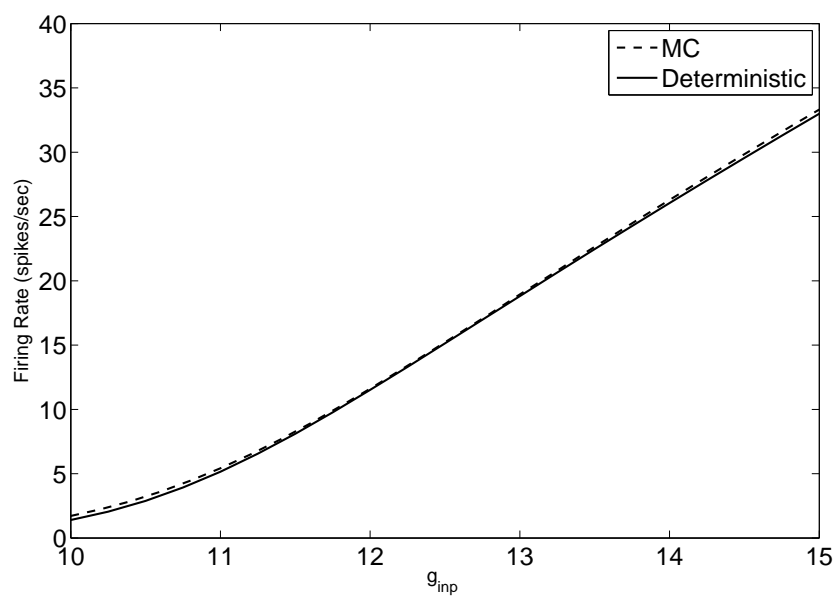

Figure 1. Evolution on time of the firing rate for Case A. Comparison between Monte Carlo and deterministic simulations.

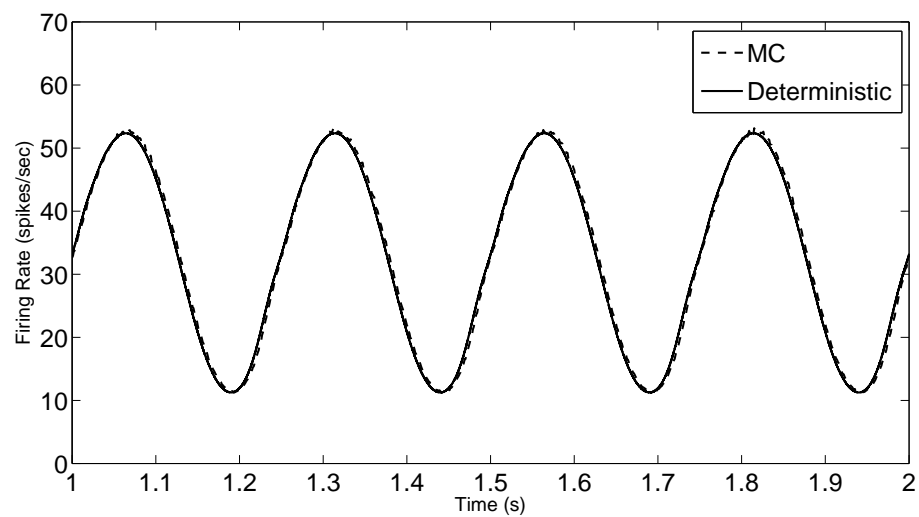

Figure 2. Evolution on time of the firing rate for Case B1. Comparison between Monte Carlo and deterministic simulations. 


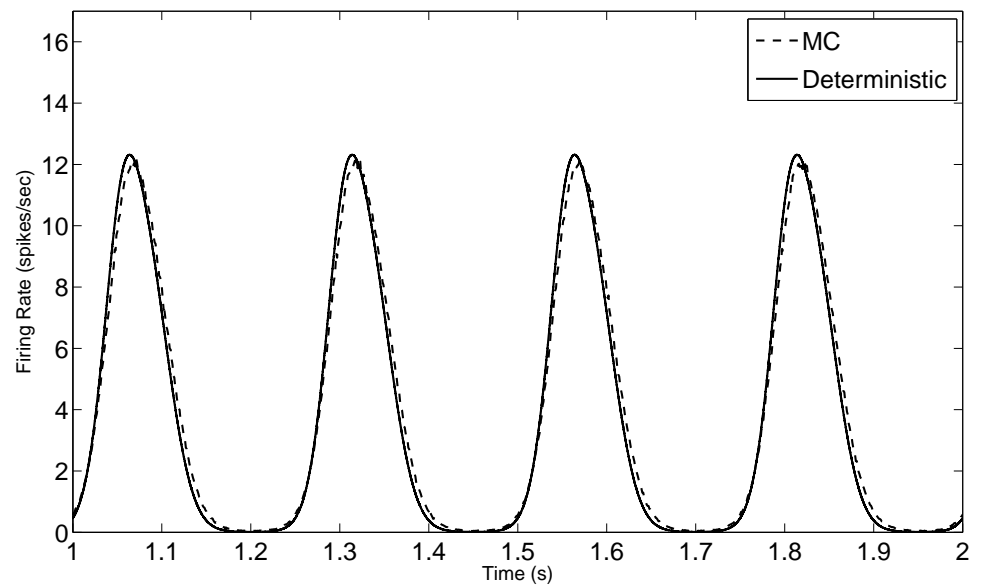

Figure 3. Evolution on time of the firing rate for Case B2. Comparison between Monte Carlo and deterministic simulations.

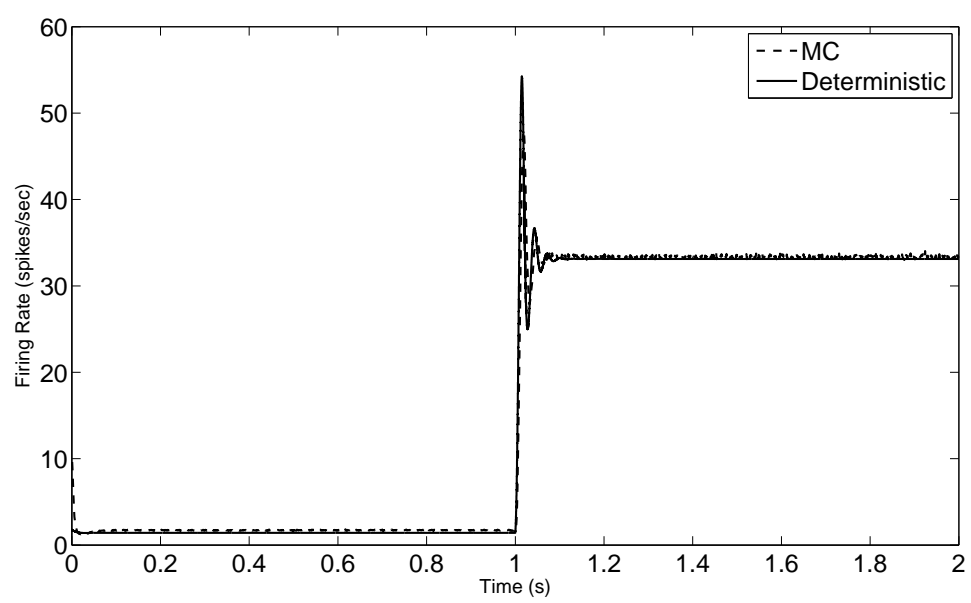

Figure 4. Evolution on time of the firing rate for Case C. Comparison between Monte Carlo and deterministic simulations. 

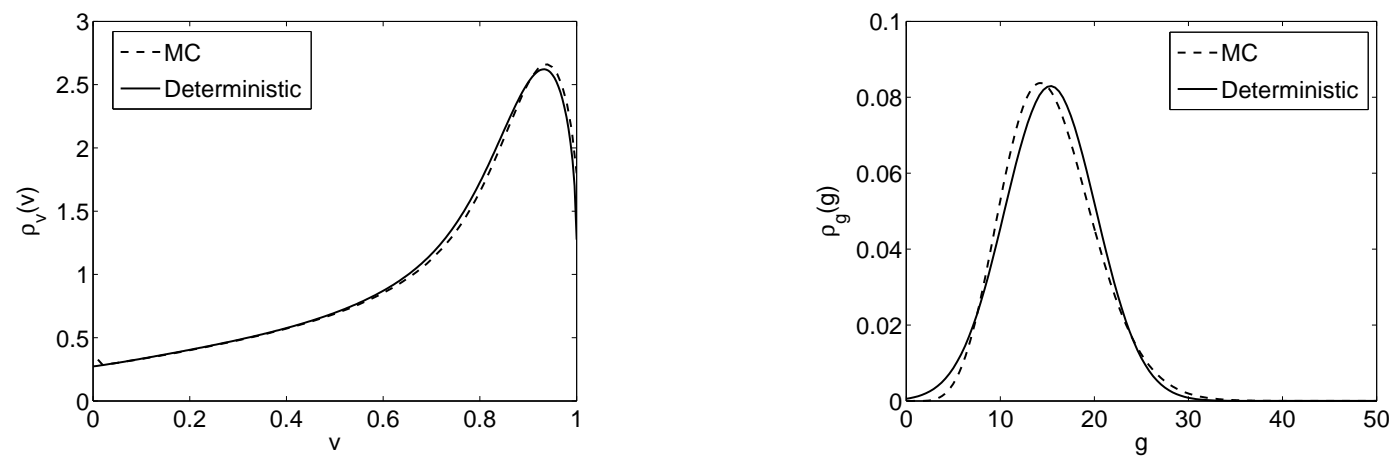

Figure 5. Comparison between Monte Carlo and deterministic simulations for the stationary distribution functions $\rho_{v}$ and $\rho_{g}$ in the Case $A$ with $\mathrm{A}=1400$.
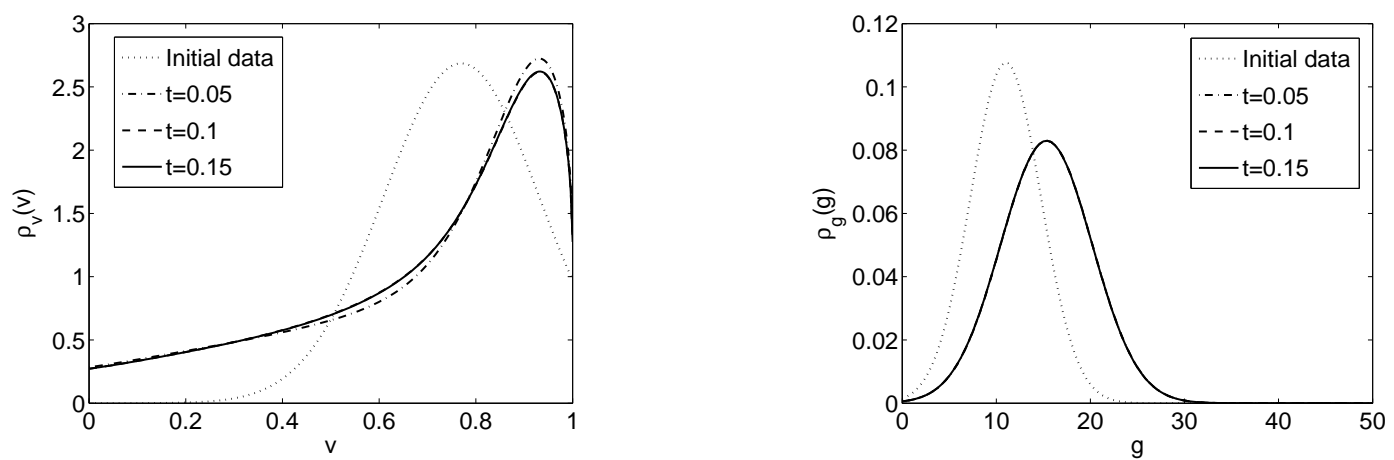

FiguRE 6. Deterministic simulations for the evolution on time of the distribution functions $\rho_{v}$ and $\rho_{g}$ in the Case $A$ with $\mathrm{A}=1400$ in four different instants: $t=0 \mathrm{~s}, t=0.005 \mathrm{~s}, t=0.1 \mathrm{~s}$ and $t=0.15 \mathrm{~s}$.

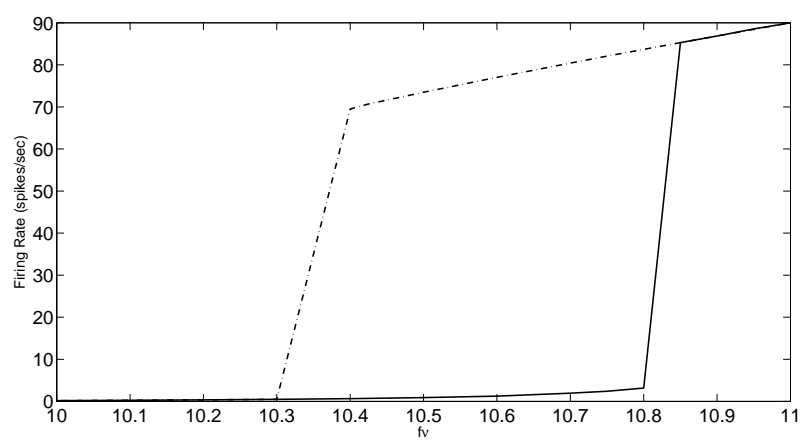

Figure 7. Stationary firing branches in Case $A$ where $f_{E}=$ $1 / 200 \mathrm{~s}, S_{E E}=0.2, N_{E}=200$ and $\sigma_{E}=2 \mathrm{~ms}$. 

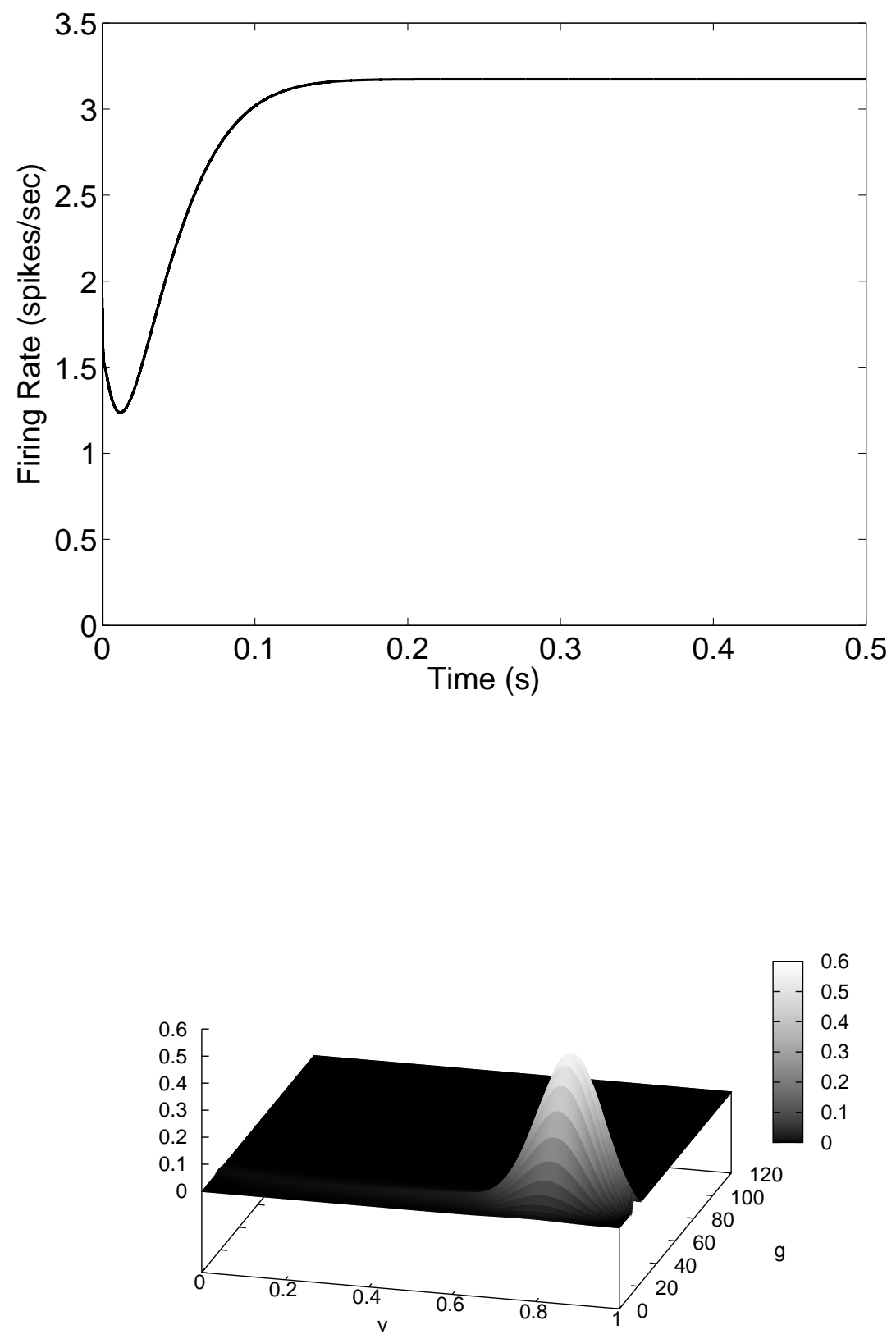

FiguRE 8. Evolution on time of the firing rate and stationary distribution function for the lower branch in Case $A$ where $f_{E}=$ $1 / 200 s, S_{E E}=0.2, N_{E}=200, \sigma_{E}=2 \mathrm{~ms}$ and $f_{E} \nu_{0 E}=10.8$. 

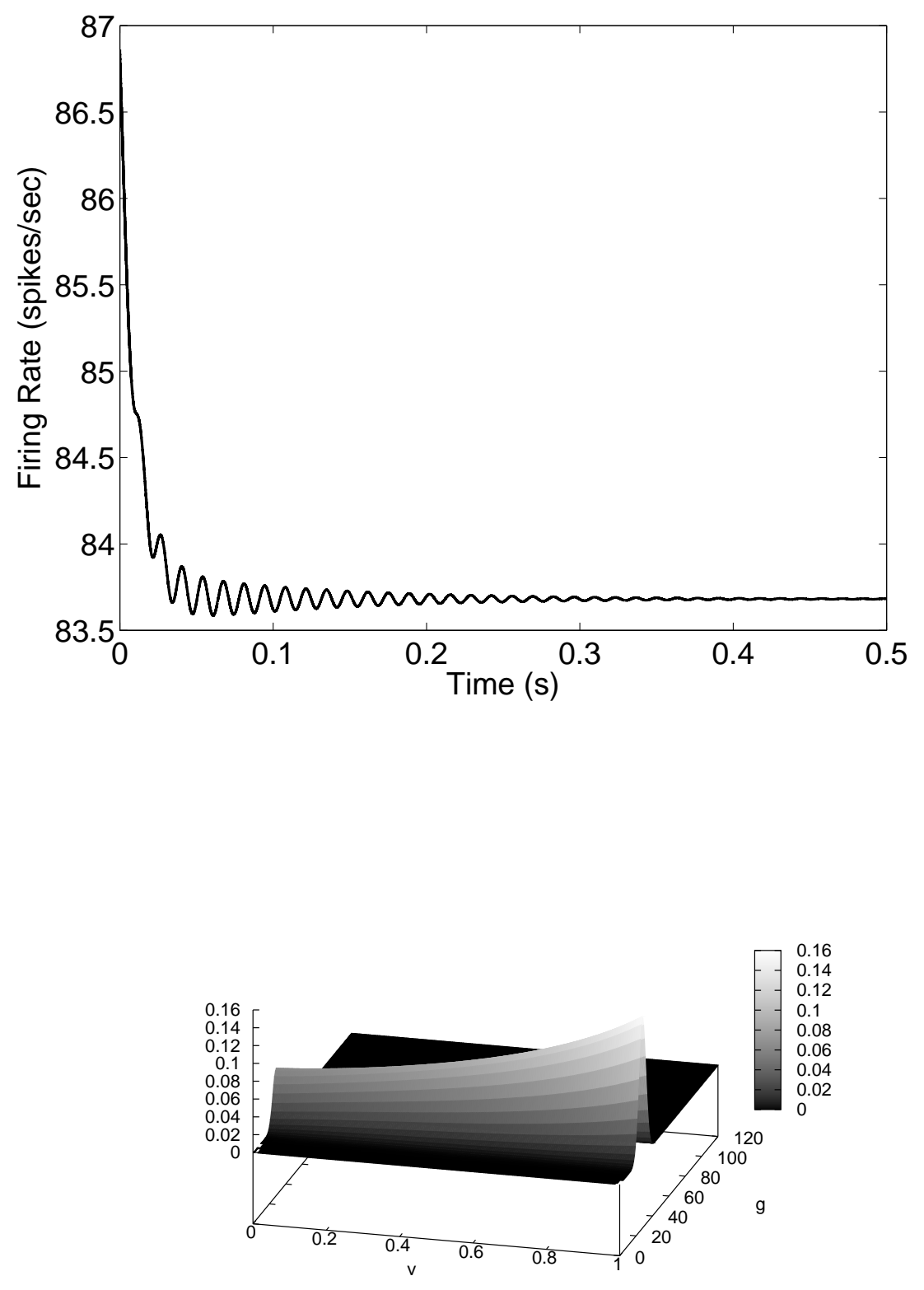

FiguRE 9. Evolution on time of the firing rate and stationary distribution function for the upper branch in Case $A$ where $f_{E}=$ $1 / 200 s, S_{E E}=0.2, N_{E}=200, \sigma_{E}=2 \mathrm{~ms}$ and $f_{E} \nu_{0 E}=10.8$. 


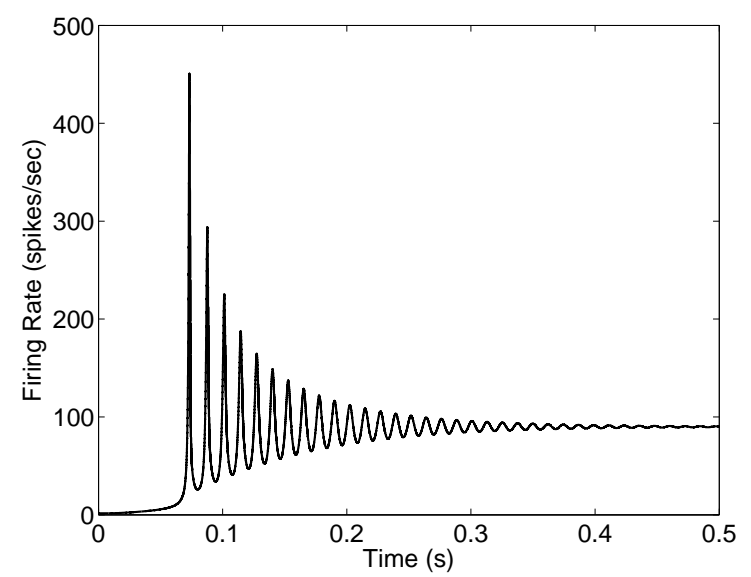

Figure 10. Evolution on time of the firing rate in the Case $A$ where $f_{E}=1 / 200 s, S_{E E}=0.2, N_{E}=200, \sigma_{E}=2 \mathrm{~ms}$ and $f_{E} \nu_{0 E}=11$.
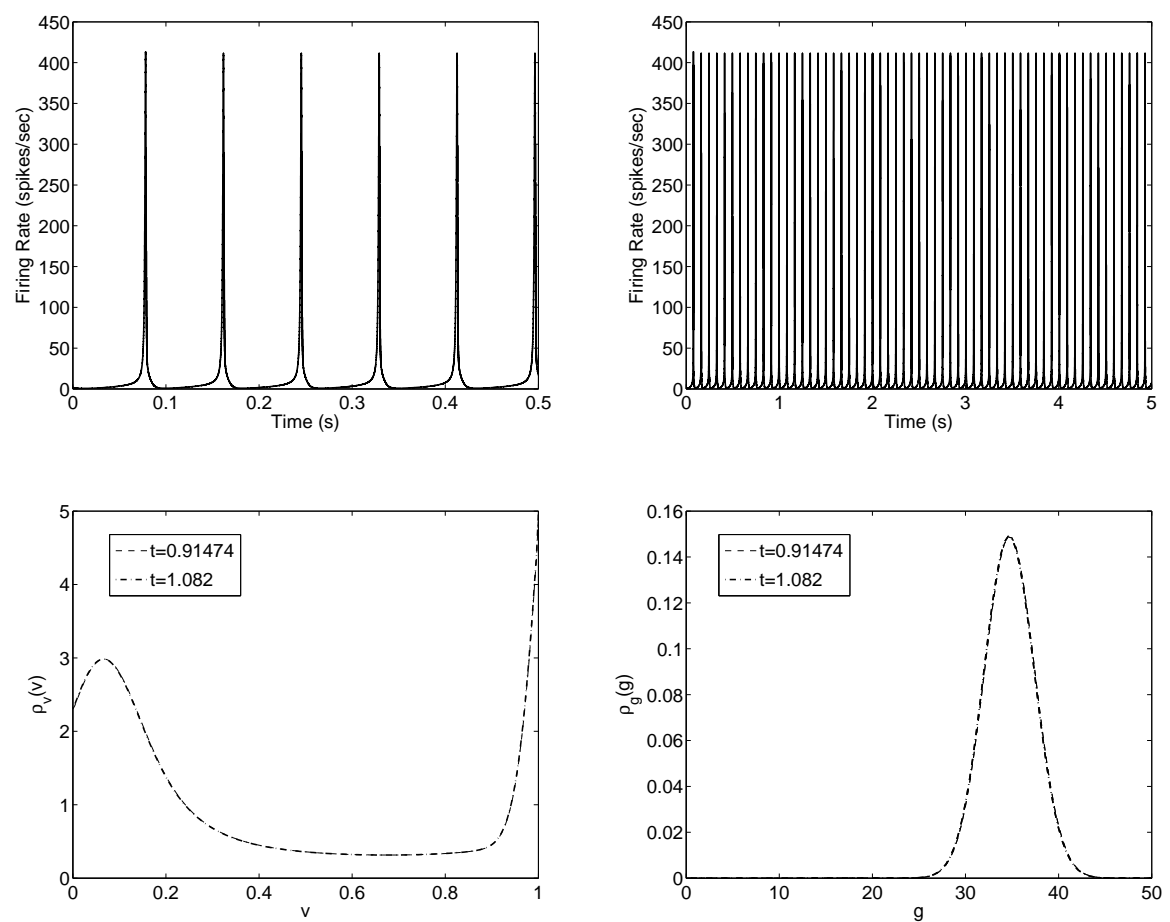

Figure 11. For Case $A$ with $S_{E E}=0.15, N_{E}=500, f_{E}=0.002 \mathrm{~s}$ and $f_{E} \nu_{0 E}=12$ the evolution on time of the firing rate (top) and the distribution functions $\rho_{v}$ (left bottom) and $\rho_{g}$ (right bottom) in two different time: $t=0.91474 \mathrm{~s}$ and $t=1.082 \mathrm{~s}$. 

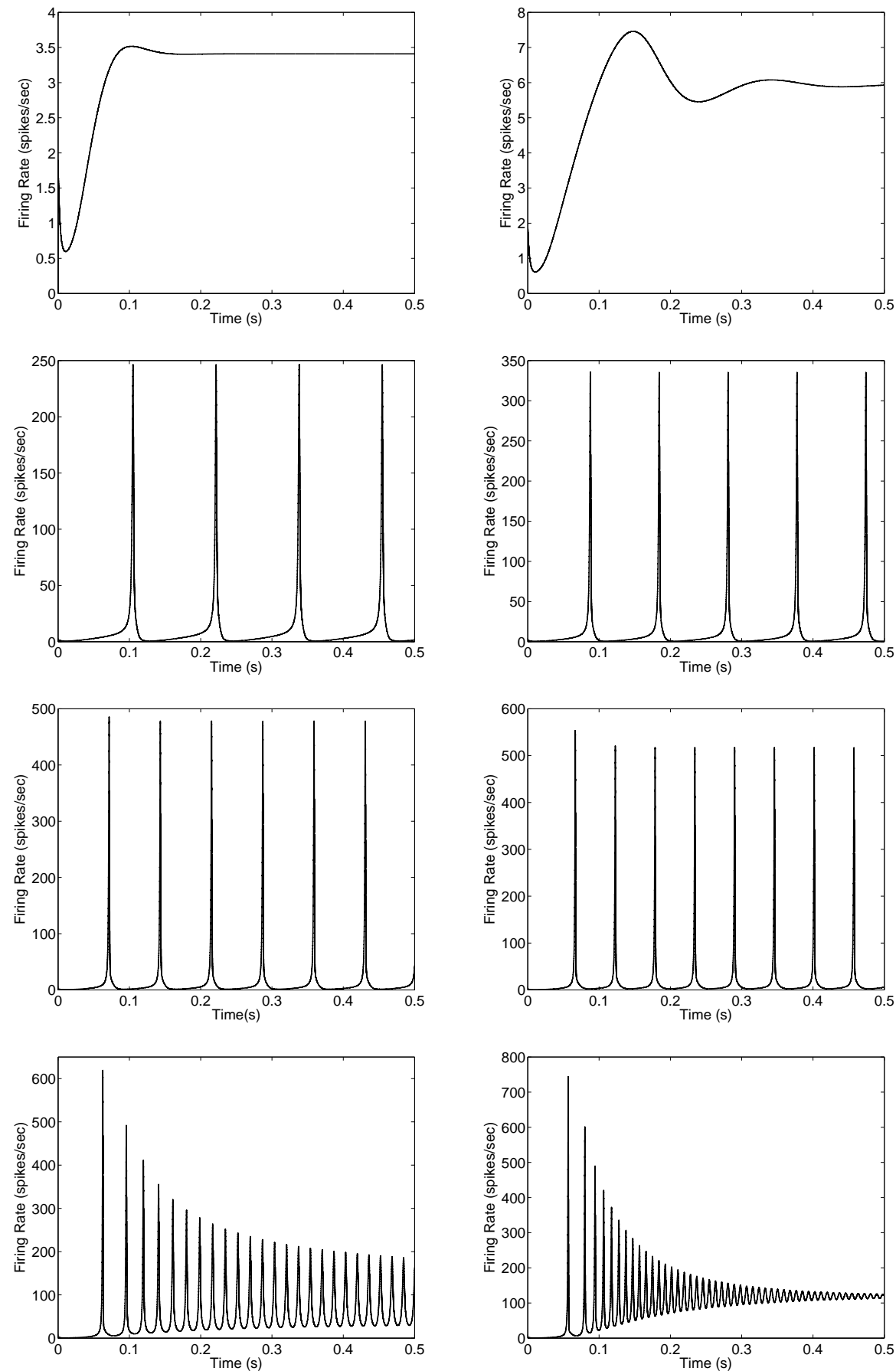

FiguRE 12. Evolution on time of the firing rates for different values of $S_{E E}$ for Case $A$ with constant: $N_{E}=500, f_{E}=0.002 \mathrm{~s}$ and $f_{E} \nu_{0 E}=12$.

From top to bottom and left to right: $S_{E E}=0.1, S_{E E}=0.12$, $S_{E E}=0.13, S_{E E}=0.14, S_{E E}=0.16, S_{E E}=0.17, S_{E E}=0.18$ and $S_{E E}=0.2$. 

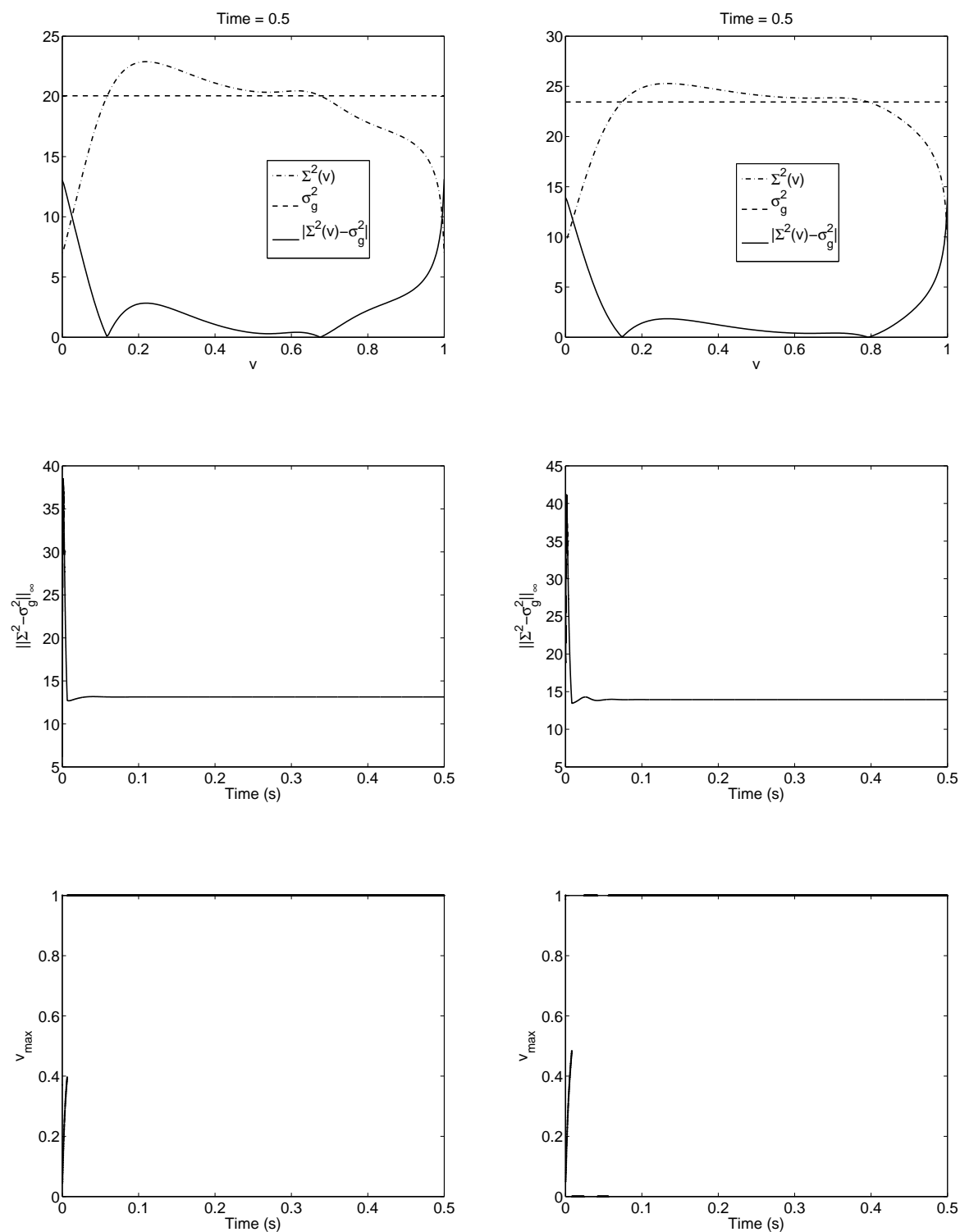

Figure 13. For Case $A$ validation of the closure (10).

Left: $A=1200$. Right: $A=1400$.

Top: $\Sigma^{2}(v), \sigma_{g}^{2}$ and $\left|\Sigma^{2}(v)-\sigma_{g}^{2}\right|$ as functions of $v$ for the stationary solution, which was reached before $0.5 \mathrm{~s}$.

Middle: Evolution on time of the $L_{\infty}$-norm of $\Sigma^{2}(v)-\sigma_{g}^{2}$.

Bottom: Maximum value of $v$ where $\left|\Sigma^{2}(v)-\sigma_{g}^{2}\right|$ reaches the value $\left\|\Sigma^{2}-\sigma_{g}^{2}\right\|_{\infty}$ 

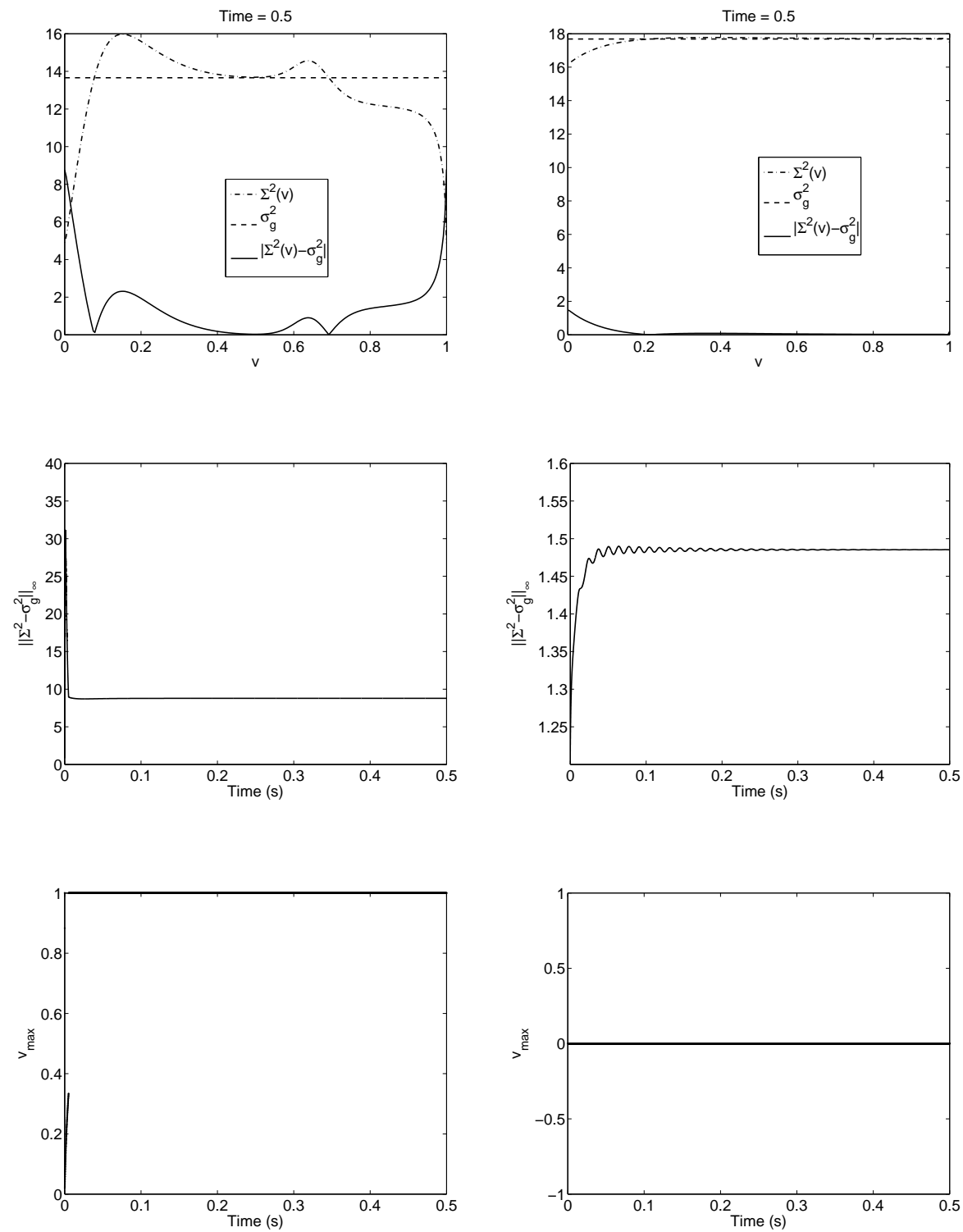

Figure 14. For Case $A$ where $f_{E}=1 / 200 s, S_{E E}=0.2, N_{E}=$ 200, $\sigma_{E}=2 \mathrm{~ms}$ and $f_{E} \nu_{0 E}=10.8$ validation of the closure (10). Left: Lower branch. Right: Upper branch.

Top: $\Sigma^{2}(v), \sigma_{g}^{2}$ and $\left|\Sigma^{2}(v)-\sigma_{g}^{2}\right|$ as functions of $v$ for the stationary solution.

Middle: Evolution on time of the $L_{\infty}$-norm of $\Sigma^{2}(v)-\sigma_{g}^{2}$.

Bottom: Maximum value of $v$ where $\left|\Sigma^{2}(v)-\sigma_{g}^{2}\right|$ reaches the value $\left\|\Sigma^{2}-\sigma_{g}^{2}\right\|_{\infty}$ 
María J. Cáceres

Departamento de Matemática Aplicada

UNIVERSIDAD DE GRANADA

18071 Granada, Spain

E-mail address: caceresg@ugr.es

José A. Carrillo

ICREA ANd Departament DE Matemàtiques

Universitat Autònoma de Barcelona

E-08193 - Bellaterra, Spain

E-mail address: carrillo@mat.uab.cat

Louis TaO

CENTER For Bioinformatics

National Laboratory of Protein Engineering

AND

Plant Genetics Engineering

College of Life Science

Peking University

100871 Beijing, P. R. China

E-mail address: taolt@cbi.pku.edu.cn 